The Astronomical JouRnAL, 118:3049-3060, 1999 December

(C) 1999. The American Astronomical Society. All rights reserved. Printed in U.S.A.

\title{
THE YARKOVSKY SEASONAL EFFECT ON ASTEROIDAL FRAGMENTS: A NONLINEARIZED THEORY FOR SPHERICAL BODIES
}

\author{
D. VOKROUHLICKÝ \\ Astronomical Institute, Charles University, V Holešovičkách 2, CZ-180 00, Prague 8, The Czech Republic \\ AND \\ P. FARINELla \\ Dipartimento di Astronomia, Università di Trieste, via Tiepolo 11, I-34131 Trieste, Italy \\ Received 1999 July 12; accepted 1999 August 16
}

\begin{abstract}
The long-term orbital evolution of meteoroids and small asteroids in the size range up to several kilometers is affected by the "seasonal" Yarkovsky force, caused by radiation pressure recoil on spinning bodies heated by solar radiation to different temperatures at different latitudes on their surfaces. This effect leads to a draglike, secular semimajor-axis decay, which may inject the bodies into chaotic zones associated with mean motion and secular resonances and eventually deliver them to near-Earth space. To model the Yarkovsky force, two kinds of simplifying assumptions have been frequently made: (1) a linearization in the ratio between temperature variation and average temperature and in the orbital eccentricity and (2) a plane-parallel geometry, that is, body sizes larger than the penetration depth of the seasonal thermal wave (typically, several meters). In a previous paper, we developed a new nonlinearized theory, and here we also remove the assumption of plane-parallel geometry and extend our theory to the more general spherical case, valid for all body sizes, by means of a new numerical approach. We also revise the linear theory, obtaining a fully analytical literal solution, which is well suited to develop analytical and semianalytical secular perturbation theories and allows us to perform a detailed comparison with the results of the more accurate numerical model. We find that the accuracy of the linear theory is relatively good $(20 \%$ or better) for near-circular orbits. Although the temperature variations grow with the orbital eccentricity, we show that the linear theory can still predict the averaged drift rates of the mean orbital elements up to eccentricities of $0.4-0.5$.
\end{abstract}

Key words: meteors, meteoroids - minor planets, asteroids

\section{INTRODUCTION}

The so-called Yarkovsky effect is a subtle nongravitational force on small orbiting bodies discovered long ago (Öpik 1951; Radzievskii 1952; Burns, Lamy, \& Soter 1979), which has become a subject of active research in recent times after its role in the delivery of small asteroidal fragments to near-Earth space was convincingly demonstrated (Rubincam 1995, 1998; Farinella, Vokrouhlický, \& Hartmann 1998; Vokrouhlický \& Farinella 1998a; Farinella \& Vokrouhlický 1999). The original, or "diurnal," component of the effect is due to recoil from radiation pressure due to differential thermal emission between the morning and afternoon hemispheres of a rotating particle; it causes a secular semimajor-axis effect whose sign depends on the sense of rotation and which is larger for small obliquities between the spin axis and the perpendicular to the orbital plane. More recently, following related work on the dynamics of artificial Earth satellites (Rubincam 1987; Vokrouhlický \& Farinella 1997), it has been pointed out that a "seasonal" component of the effect is also to be expected, because of the temperature asymmetry between the winter and summer hemispheres; this seasonal Yarkovsky effect is maximum at large obliquities and always causes a secular, draglike semimajor-axis decay (Rubincam 1995, 1998; Vokrouhlický \& Farinella 1998b).

The two components of the Yarkovsky effect correspond to two limiting cases, which can be found by comparing the rotation period $T_{\text {rot }}$ with the thermal relaxation time $T_{\text {rel }}$, namely, the time required to reemit the thermal energy absorbed from solar radiation during a rotational cycle. The diurnal effect is the dominant one when the corre- sponding ratio $T_{\text {rot }} / T_{\text {rel }}$ is large (as it appears to be for slow rotation), so that the surface temperature has significant gradients both in longitude and in latitude. If the relaxation time were exactly zero, the absorbed radiation would be reemitted instantaneously; in this case, the surface temperature profile has a characteristic symmetric behavior on the illuminated part of the body (in radiometry this approximation has been often called the "nonrotating standard model"; see, e.g., Spencer, Lebofsky, \& Sykes 1989). When the relaxation time is not exactly zero but very small, the picture is roughly the same, but the temperature peak is slightly shifted in the sense of the body's rotation. The recoil force due to the thermal emission is oriented approximately toward the point of maximum temperature, that is, opposite to the external radiation source (the Sun), but with a small shift in the sense of the body's rotation (see Vokrouhlický 1998). This gives rise to an along-track component of the force, resulting in a secular change of the semimajor axis.

The seasonal component of the Yarkovsky effect is important when the thermal relaxation time is much longer than the rotational period of the body. In this case, there is almost no longitude dependence of the surface temperature, only a kind of latitude stratification, since it takes so long to reemit the absorbed radiation that all the surface elements on a given latitude ring "forget" their individual histories (in radiometry this approximation has been often called the "fast-rotating standard model"; see, e.g., Spencer et al. 1989). For symmetry reasons, the resulting thermal recoil force is aligned with the spin axis, but the peak force occurs after the solstices by a time lag depending on the ratio between the relaxation time and the orbital period. It is this 
time lag that results in a draglike force component (Rubincam 1995, 1998; Vokrouhlický \& Farinella 1998b; Vokrouhlický 1999). Note that if the body's sense of rotation changes in a random fashion, e.g., as a result of collisions from other orbiting particles, the seasonal effect is favored over the diurnal one as a cause of semimajor-axis drift, because the former one always provokes orbit decay, whereas the latter results in a kind of random walk.

To model these subtle effects in a quantitative way, several approximations have been frequently used, such as (1) considering near-circular orbits (small eccentricities), (2) assuming that the temperature variations on the surface are always much smaller than the average temperature, and (3) taking a very simple, one-dimensional geometry (planeparallel case), that is, assuming that the body's size is much larger than the depth of the layer where the external illumination causes significant temperature changes. In a recent paper (Vokrouhlický \& Farinella 1998b), we developed a theory in which approximations 1 and 2 have been removed from the treatment of the seasonal effect and have shown that this leads to some interesting discrepancies with respect to the simpler case, linearized with respect to the amplitude of the temperature variations. However, approximation 3 means that (for material properties typical of rocky bodies) these results are meaningful only for objects larger than about $10 \mathrm{~m}$. Therefore, here we develop a new numerical approach to the problem in which approximation 3 is also removed, so that the results can be applied with confidence to smaller bodies. This is important for applications related to meteorite transport from the asteroid belt (Farinella et al. 1998), because the preatmospheric sizes of most meteorites range from about $10 \mathrm{~cm}$ to $10 \mathrm{~m}$. We also revise the analytical treatment of the seasonal effect in the linearized approach by removing any truncation in the orbital eccentricity development. This enables us to derive fairly general analytical formulae for the long-term variations of all five mean orbital elements.

The remainder of this paper is structured as follows: In $\S 2$, we develop the new theory and describe the corresponding numerical technique. In $\S 3$, we discuss a number of examples to compare the results from the new theory with those from the previous ones using approximations 1 to 3 above and identify the most interesting new findings related to the astronomical applications of the theory. Section 4 provides a summary of our main conclusions. In Appendix $\mathrm{A}$, we give a brief discussion of the numerical technique used to solve the nonlinearized heat diffusion problem as formulated in $\S 2$. In Appendix B, we illustrate a simplified analytical solution based on approximation 2 alone, which is useful for comparison purposes and well suited for analytical perturbation approaches to assess the long-term orbital effects.

\section{THEORY}

The problem of heat conduction in a passive solid body is described by the parabolic diffusion equation

$$
\bar{\rho} C \frac{\partial T}{\partial t}=K \nabla^{2} T,
$$

which expresses energy conservation for each volume element of the (rigid) body. The goal is to find the temperature, $T$, distribution throughout the body at any time $t$ (here $\nabla^{2}$ is the Laplace operator, $\bar{\rho}$ the density, $C$ the specific heat, and $K$ the thermal conductivity). The material properties will be assumed to be constant. An extension to cases in which the material parameters (especially the thermal conductivity $K$ ) are temperature dependent or the body is not homogeneous might be interesting, but they are beyond the capability of the method used in this paper. For instance, we refer to Vokrouhlický \& Brož (1999) for a linearized theory of the seasonal Yarkovsky effect on a composite body with a low-conductivity surface layer and a higher conductivity core.

Conservation of energy at the body's surface provides the additional equation

$$
\epsilon \sigma T^{4}+K\left(\boldsymbol{n} \cdot \frac{\partial T}{\partial \boldsymbol{r}}\right)=(1-A) \mathscr{E},
$$

where $\epsilon$ is the surface emissivity; $\sigma$, the Stefan-Boltzmann constant; $\boldsymbol{n}$, the surface normal unit vector; $A$, the surface albedo; and $\mathscr{E}$, the external energy flux (in our case, solar radiation). Equation (2) expresses the balance between the incoming energy (right-hand side) and the sum of the thermally reradiated energy (first term on the left-hand side) and the thermal energy conducted from the interior of the body (second term on the left-hand side). This equation plays the role of a boundary condition for the heat diffusion problem. A second such condition is the regularity of the temperature distribution throughout the body.

In what follows, we shall adopt some simplifying assumptions that will allow us to treat the problem efficiently. First, we propose to study a spherical body that spins fast enough that the temperature is constant at any given "latitude" (i.e., we deal with only the seasonal component of the Yarkovsky effect, as defined in $\S 1$ ). As a result, the temperature depends only on the radial distance from the center $r$ and the colatitude $\theta$ (hereafter we shall use the parameter $\mu=\cos \theta$ instead of $\theta$ ). Because of the fast rotation, we can average equations (1) and (2) over one rotational cycle. Therefore, the right-hand side of equation (2) corresponds to the total energy flux emerging through the surface ring in the latitude range $(\mu, \mu+d \mu)$. Moreover, we shall rewrite our basic equations (1) and (2) by rescaling the physical parameters appearing in them with appropriate units of length and time. As we are dealing with the seasonal Yarkovsky effect, a natural scale of length is the penetration depth $l_{s}$ of the thermal wave during one orbital revolution, that is,

$$
l_{s}=\sqrt{\frac{K}{\bar{\rho} C n}} .
$$

Here $n$ is the mean motion of the body's orbit around the Sun. Our scaled radial coordinate is then $r^{\prime}=r / l_{s}$. In a similar way, instead of time $t$ we shall use the complex nondimensional variable $\zeta$, defined by

$$
\zeta=\exp (i n t)
$$

where $i=(-1)^{1 / 2}$. The origin of time in equation (4) is supposed to coincide with an arbitrary pericenter passage. Then it is easy to show that all the physical parameters appearing in equations (1) and (2) collapse into a single thermal parameter $\Theta$, given by

$$
\Theta=\frac{\Gamma \sqrt{n}}{\epsilon \sigma T_{*}^{3}}
$$


(an additional, "hidden" parameter is the radius $R$ of the body, expressed in thermal length units $l_{s}$ ). Here $\Gamma=$ $(K \bar{\rho} C)^{1 / 2}$ is the thermal inertia and $T_{*}$ is an effective subsolar temperature (at a distance from the Sun equal to the semimajor axis corresponding to $n$ ) defined by

$$
\epsilon \sigma T_{*}^{4}=(1-A) \mathscr{E}_{*},
$$

where $\mathscr{E}_{*}$ is the solar radiation flux at the given heliocentric distance. To convert equations (1) and (2) into a nondimensional form, we also normalize the temperature $T$ by $T_{*}$ and the energy flux $\mathscr{E}$ by $\mathscr{E}_{*}$. The scaled variables will be denoted by primes: $T^{\prime}=T / T_{*}$ and $\mathscr{E}^{\prime}=\mathscr{E} / \mathscr{E}_{*}$. Note that, following Spencer et al. (1989), we prefer to use $T_{*}$ as a scaling factor, rather than the mean temperature $\bar{T}$ (see eq. [20] below, adopted by Rubincam 1995, 1998).

With the assumptions and definitions listed above, the heat diffusion equation (1) can be rewritten in the following form:

$$
i r^{\prime 2} \zeta \frac{\partial}{\partial \zeta} T^{\prime}\left(r^{\prime}, \mu ; \zeta\right)=\Lambda\left(r^{\prime}, \mu\right) T^{\prime}\left(r^{\prime}, \mu ; \zeta\right)
$$

with the operator $\Lambda\left(r^{\prime}, \mu\right)$ defined as

$$
\Lambda\left(r^{\prime}, \mu\right)=\frac{\partial}{\partial r^{\prime}}\left(r^{\prime 2} \frac{\partial}{\partial r^{\prime}}\right)+\frac{\partial}{\partial \mu}\left[\left(1-\mu^{2}\right) \frac{\partial}{\partial \mu}\right],
$$

whereas the boundary condition (eq. [2]) at the surface of the body (that is, at $r^{\prime}=R^{\prime}=R / l_{s}$ ) now reads

$$
T^{\prime 4}\left(R^{\prime}, \mu ; \zeta\right)+\Theta\left(\frac{\partial T^{\prime}}{\partial r^{\prime}}\right)\left(R^{\prime}, \mu ; \zeta\right)=\mathscr{E}(\mu ; \zeta) .
$$

Equations (7) and (9) represent a complicated, nonlinear problem, as is well known from the literature (e.g., Peterson 1976; Rubincam 1995; Vokrouhlický \& Farinella 1998b). However, it is important to note that the only real obstacle to obtain an analytical solution of the problem lies in the boundary constraint (eq. [9]), where the nonlinearity in the temperature variable occurs (first term of the left-hand side). Without any loss of generality, we can still derive a complete analytical solution of the Fourier equation (7). In particular, we may assume a mixed Fourier-Legendre development

$$
\begin{aligned}
T^{\prime}\left(r^{\prime}, \mu ; \zeta\right) & =\sum_{k \in \mathbf{Z}} t_{k}\left(r^{\prime}, \mu\right) \zeta^{k} \\
& =\sum_{k \in \mathbf{Z}} \sum_{l \geq 0} \tau_{k l}\left(r^{\prime}\right) P_{l}(\mu) \zeta^{k}
\end{aligned}
$$

Here the symbol $\mathbf{Z}$ means the summation is over all integers, and $P_{l}(\mu)$ are the Legendre polynomials. The functions $\tau_{k l}\left(r^{\prime}\right)$, giving the radial profile of the temperature in the body, read

$$
\begin{aligned}
& \tau_{0 l}\left(r^{\prime}\right)=c_{0 l} r^{\prime l}, \\
& \tau_{k l}\left(r^{\prime}\right)=c_{k l} j_{l}\left(\sqrt{-i k r^{\prime}}\right),
\end{aligned}
$$

where $j_{l}(z)$ are spherical Bessel functions and $c_{0 l}$ and $c_{k l}$ are constants (we assume $k \neq 0$ in eq. [11b]). These constants should be determined from the boundary condition, equation (9). In what follows, we shall also use another set of constants that determine the solution, namely, $C_{k l}=\tau_{k l}\left(R^{\prime}\right)$ $(k \in \mathbf{Z}$ and $l \geq 0)$. Since an appropriate representation of the energy source term $\mathscr{E}^{\prime}(\mu ; \zeta)$ in the right-hand side of (eq.
[9]) is very important in the procedure to obtain the set of constants $C_{k l}$, we briefly comment on this problem below.

The piecewise definition of $\mathscr{E}^{\prime}$ reads

$$
\begin{aligned}
\mathscr{E}^{\prime}(\mu ; \zeta)= & \frac{\psi(\zeta)}{\pi}\left[\mu \phi_{*}(\mu ; \zeta) \cos \theta_{0}(\zeta)\right. \\
& \left.+\sqrt{1-\mu^{2}} \sin \phi_{*}(\mu ; \zeta) \sin \theta_{0}(\zeta)\right],
\end{aligned}
$$

(e.g., Vokrouhlický \& Farinella 1998b), where we have introduced the function

$$
\psi(\zeta)=\left[\frac{1+e \cos v(\zeta)}{1-e^{2}}\right]^{2}
$$

$[v(\zeta)$ is the true anomaly along the orbit]. The auxiliary angle $\phi_{*}$ is defined by

$$
\cos \phi_{*}(\mu ; \zeta)= \begin{cases}-1 & \text { for } \theta<\theta_{-}, \\ -\frac{\mu}{\sqrt{1-\mu^{2}}} \cot \theta_{0}(\zeta) & \text { for } \theta \in\left(\theta_{-}, \theta_{+}\right), \\ 1 & \text { for } \theta>\theta_{+},\end{cases}
$$

and $\theta_{ \pm}=(\pi / 2) \pm \theta_{0}(\zeta)$. The angle $\theta_{0}(\zeta)$ between the solar direction and the body's spin axis is given by

$$
\cos \theta_{0}(\zeta)=-\left[s_{P} \cos v(\zeta)+s_{Q} \sin v(\zeta)\right],
$$

where $s_{P}=\boldsymbol{s} \cdot \boldsymbol{P}$ and $s_{Q}=\boldsymbol{s} \cdot \boldsymbol{Q}$ are the projections of the spin-axis unit vector $s$ onto the directions of the orbital pericenter $\boldsymbol{P}$ and of $\boldsymbol{Q}=\boldsymbol{N} \times \boldsymbol{P}(\boldsymbol{N}$ being the unit vector parallel to the orbital angular momentum). Although several authors have attempted to obtain an elliptic expansion of the insolation function in equations (12), (13), and (14) analytically (e.g., Rubincam 1994; Vokrouhlický 1998, unpublished notes), the result is quite cumbersome, especially for high-eccentricity orbits. Therefore, as we did for the temperature (eq. [10]), we use a Fourier-Legendre expansion,

$$
\mathscr{E}^{\prime}(\mu ; \zeta)=\sum_{k \in \mathbf{Z}} \varepsilon_{k}(\mu) \zeta^{k}=\sum_{k \in \mathbf{Z}} \sum_{l \geq 0} \varepsilon_{k l} P_{l}(\mu) \zeta^{k},
$$

and compute the coefficients $\varepsilon_{k l}$ by a direct numerical quadrature. In particular, we have

$$
\begin{aligned}
\varepsilon_{k}(\mu) & =\frac{1}{2 \pi i} \oint \frac{d \zeta}{\zeta} \mathscr{E}^{\prime}(\mu ; \zeta) \zeta^{-k}, \\
\varepsilon_{k l} & =\frac{2 l+1}{2} \int_{-1}^{1} d \mu \varepsilon_{k}(\mu) P_{l}(\mu),
\end{aligned}
$$

with the integration in equation (16a) performed over the unit circle in the complex plane.

Given the temperature $T^{\prime}$ expressed in terms of the Fourier-Legendre series (eq. [10]), we may similarly develop its fourth power $T^{\prime 4}$, that is,

$$
T^{\prime 4}\left(R^{\prime}, \mu ; \zeta\right)=\sum_{k \in \mathbf{Z}} \sum_{l \geq 0} \sigma_{k l}\left(C_{p q}\right) P_{l}(\mu) \zeta^{k} .
$$

Obviously, the amplitudes $\sigma_{k l}$ of this development depend on the corresponding amplitudes $C_{p q}$ in equation (11). Although this dependence is quite complicated if it has to be expressed analytically, we shall not need it in our numerical scheme (for details, see Appendix A). Rather, after having 
derived the coefficients $C_{p q}$ at some stage in the solution, we compute the $T^{\prime 4}$ function on the surface and determine the coefficients $\sigma_{k l}$ numerically by using equations (16a) and (16b) where $T^{\prime 4}$ replaces $\mathscr{E}^{\prime}$.

The orthogonality of the Fourier and Legendre series then results in the following decomposition of the boundary constraint (eq. [9]) into individual modes (it is sufficient to assume $k, l \geq 1$ here):

$$
\begin{array}{r}
\sigma_{00}\left(C_{p q}\right)=\varepsilon_{00,} \\
\sigma_{0 l}\left(C_{p q}\right)+l \frac{\Theta}{R^{\prime}} C_{0 l}=\varepsilon_{0 l} \\
\sigma_{k l}\left(C_{p q}\right)+\psi_{l}\left(Z_{k}\right) \frac{\Theta}{R^{\prime}} C_{k l}=\varepsilon_{k l}
\end{array}
$$

( $k \neq 0$ in the last of these equations). We have introduced

$$
\psi_{l}\left(Z_{k}\right)=\left[z \frac{j_{l}^{\prime}(z)}{j_{l}(z)}\right]_{Z_{k}},
$$

with $Z_{k}=(-i k)^{1 / 2} R^{\prime}$ and the prime denoting the derivative of the Bessel function with respect to the argument $z$.

Equations (18a)-(18c) then represent a nonlinear system of algebraic equations for the coefficients $C_{k l}$. Despite its complexity, we stress that in this way the problem of solving the partial differential equations (7) and (9) has been converted into the problem of solving an infinite set of algebraic equations. Of course, in practice we shall restrict ourselves to a finite subset of these equations, since all the coefficients decrease in magnitude for increasing values of $k$ (and $l$ too). This conclusion holds for orbits of any eccentricity, but finding a suitable threshold for $k$ to represent the solution accurately enough is a matter of numerical experimentation.

A suitable iteration method, starting from the linearized approximation to the solution, which solves the system of equations (18a)-(18b), is briefly described in Appendix A. The starting solution, which is then improved by iterations, is provided by the average temperature

$$
C_{00}\left(=\bar{T}^{\prime}\right)=\eta^{-1 / 4} / \sqrt{2},
$$

with $\eta=\left(1-e^{2}\right)^{1 / 2}$ (where $e$ is the orbital eccentricity), and by the linearized solution

$$
\begin{aligned}
& C_{k 1}=-\frac{\chi_{k} \eta^{3 / 4}}{4 \sqrt{2}(1+\lambda)} \frac{A\left(X_{k}\right)+i B\left(X_{k}\right)}{C\left(X_{k}\right)+i D\left(X_{k}\right)}, \\
& C_{k l}=0 \quad \text { for } l \geq 0 \text { and } k \geq 2 .
\end{aligned}
$$

The variables of the linearized solution are introduced in Appendix B. Obviously, in practical implementations the coefficients $C_{k l}$ of an exact solution of equations (18a)-(18b) with some values of the thermal parameters and the orbitspin-axis geometry can be stored and then retrieved to be used as a seed for starting the iteration procedure in another case with similar parameter values. In this way, a lower number of iterations is usually required to attain a predefined accuracy level.

At convergence of the iteration scheme, we obtain the coefficients $C_{k l}$ with a specified accuracy (see Appendix A for more quantitative discussion). Then we can determine the surface temperature distribution by setting $r^{\prime}=R^{\prime}$ in equation (10) at any latitude $\mu$ and any time $\zeta$, that is, $T^{\prime}(\mu ; \zeta)=\sum C_{k l} P_{l}(\mu) \zeta^{k}$. This result can be used in a number of applications, for instance, in the thermal models used in asteroid radiometry or to study the temperature distribution inside the body (e.g., with the aim of obtaining the thermal stresses in the interior). Here we restrict ourselves to the orbital perturbations associated with the Yarkovsky effect. The thermal recoil force per unit mass of the body can be obtained by a numerical quadrature of the equation

$$
\begin{aligned}
\boldsymbol{a}_{\mathrm{th}}(\zeta) & =-\frac{4 s}{3}(1-A) \rho \int_{-1}^{1} d \mu \mu T^{\prime 4}\left(R^{\prime}, \mu ; \zeta\right) \\
& =-\frac{8 s}{9}(1-A) \rho \sum_{k \in \mathbf{Z}} \sigma_{k 1}\left(C_{p q}\right) \zeta^{k},
\end{aligned}
$$

where $s$ is the unit vector in the direction of the spin axis and the quantity $\rho=\pi R^{2} \mathscr{E}_{*} / m c$ is the usual radiation force factor ( $m$ is the body's mass and $c$ is the velocity of light).

\section{EXAMPLES AND COMPARISONS WITH SIMPLIFIED SOLUTIONS}

In this section, we are going to discuss several specific applications of the seasonal Yarkovsky force theory described $\S 2$, and in particular, we will compare the results with those from the simpler theories based on the two simplifying approximations frequently made in the previous literature: (1) the linear analytical solution (outlined here in Appendix B; see also Rubincam 1995, 1998; Vokrouhlický 1999) and (2) the nonlinearized solution for the planeparallel case (the large-body case discussed by Vokrouhlický \& Farinella 1998b).

\subsection{Circular Orbits}

First, we discuss the solution for the surface temperature distribution, as this is the principal factor determining the thermal effects. For the sake of simplicity, we assume that the body's spin axis lies in the orbital plane (obliquity $\gamma=90^{\circ}$ ) and compute the polar temperature $T_{p}^{\prime}$ at a colatitude $\mu=1$. We also assume a circular orbit, with the time origin chosen so that the irradiation of the pole is maximum at the mean anomaly $\phi=0$ (e.g., $s_{P}=-1, s_{Q}=0$ ). Figure 1 shows the polar temperature profiles for bodies of different radii $R^{\prime}$ but the same thermal parameter $\Theta=1$. The thick curve with an $\infty$ label corresponds to the plane-parallel geometry solution of Vokrouhlický \& Farinella (1998b; see also Spencer et al. 1989). When the radius of the body gets smaller, the temperature variations become less pronounced and its excursions around the mean temperature $\bar{T}^{\prime} \approx 1 /(2)^{1 / 2}$ decrease. This feature is simply due to the fact that thermal conduction for bodies with $R^{\prime}<1$ (i.e., radius $R$ smaller than the thermal wave penetration depth $l_{s}$ ) is quite efficient in averaging out the temperature excursions on the surface. Every surface element tends to "forget" its individual irradiation history and approach the temperature of the whole body. As noted by Rubincam (1998), in this case the resulting seasonal Yarkovsky force becomes smaller and smaller. We also note that the actual mean temperature over the body $\left(\tau_{00}\right)$ is slightly smaller than the linearized-theory value $1 /(2)^{1 / 2}$ (e.g., for $R^{\prime}=0.5$ we obtain $\tau_{00} \simeq 0.698$ ). This difference is due to a contribution of the higher order terms, and it was noted by Peterson (1976) in his treatment of a quadratic theory. 


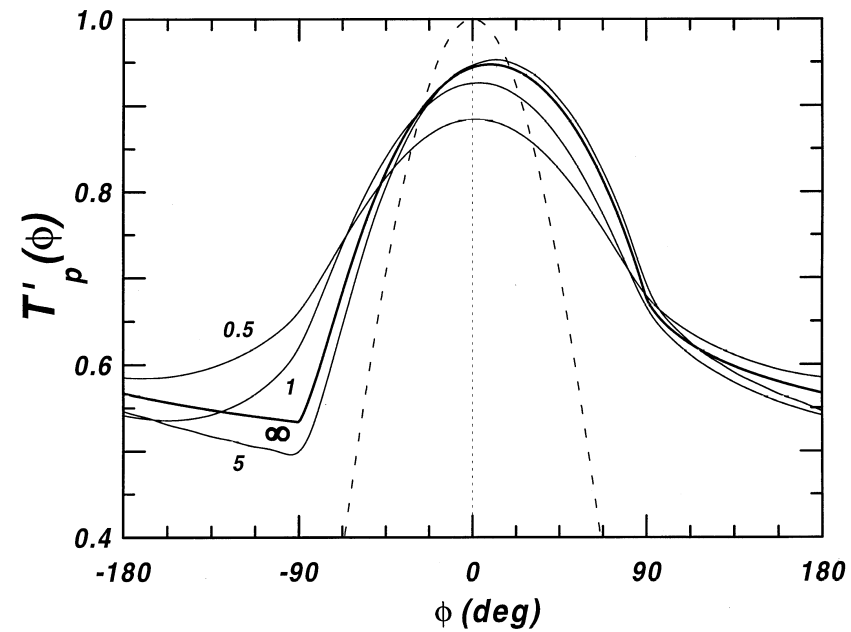

FIG. 1.-Polar temperatures of spherical bodies with different radii $R^{\prime}$, indicated by labels on the curves, along one revolution in a circular orbit. The rotation axis is assumed to lie in the orbital plane $\left(\gamma=90^{\circ}\right)$ and the thermal parameter is always fixed at $\Theta=1$. The time origin is chosen in such a way that the energy influx at the pole is given by the dashed line (here the scale is normalized at 1 at the peak of the curve). The thickest curve (label $\infty$ ) corresponds to the plane-parallel solution of Vokrouhlický \& Farinella (1998b).

A closer look at Figure 1 reveals that the previous physical scenario is an accurate description of what happens only for bodies with $R^{\prime} \leq 1$. Interestingly, intermediate-size bodies (e.g., our solution for $R^{\prime} \approx 5$ ) exhibit the opposite effect. When compared with the plane-parallel solution (thick curve), the amplitude of their temperature variations is greater by about the same amount by which the temperature peak lags behind the maximum of irradiation. This is an interesting result because the thermal penetration depth $l_{s}$ is about $2-4 \mathrm{~m}$ for stony fragments at $a \approx 2 \mathrm{AU}$, and therefore, the greatest temperature variations occur for objects $10-20 \mathrm{~m}$ in radius. The uncertainty in this latter size is due to that in $l_{s}$, and the latter comes mainly from our poor knowledge of the thermal conductivity $K$ for asteroid fragments (see, e.g., Farinella et al. 1998).

Apart from the amplitude of the temperature variations, the secular effects on the semimajor axis depend sensitively on the angle by which the surface temperature lags behind the incident solar flux. Figure 2 shows the polar temperature lag angle as a function of the thermal parameter $\Theta$ for bodies with different radii $R^{\prime}$. Our numerical nonlinear solutions (solid curves) are compared with those from the linear theory (dashed curves). We note that the linear theory always overestimates the lag angle. This result was anticipated by Rubincam (1995) in the large-body case, but apparently it is fairly general. On the other hand, the linear theory systematically underestimates the amplitude of the temperature variations. These two effects compete with each other in affecting the semimajor-axis decay rate as predicted by the linear theory. To assess which of them is stronger, we have plotted the orbit-averaged transverse component $S$ of the thermal force in Figure 3. At least for low-eccentricity orbits, this quantity is unambiguously related to the semimajor-axis decay rate [since $d a / d t \approx 2 S /$ $n+O(e)$, where $n$ is the mean motion]. The results from the linear theory (see eq. [B12a] in Appendix B) are shown by dashed curves and compared with the results from the

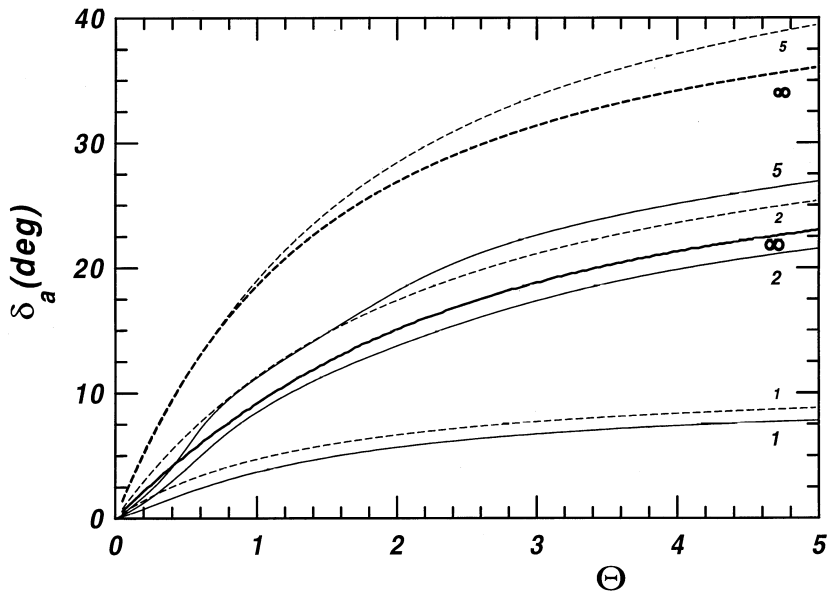

FIG. 2.- Lag angle of the polar surface temperature peak from Fig. 1 vs. the thermal parameter, $\Theta$. The linear solutions (dashed curves) systematically overestimate the lag angle when compared with the more accurate results from our numerical approach (solid curves). Labels indicate the scaled radius $R^{\prime}$ of the body.

numerical theory, shown by solid curves. In the limiting case of a plane-parallel geometry (label $\infty$ ), we have used equation (27) of Rubincam (1995) in the linear case and the numerical nonlinear results of Vokrouhlický \& Farinella (1998b). We note that the maximum discrepancy, about $20 \%$, occurs for large bodies $\left(R^{\prime} \gg 1\right)$ with $\Theta \approx 1$. For bodies of small size $\left(R^{\prime} \leq 1\right)$, the difference is less pronounced and the linear approximation matches the exact theory within a few percent. This is consistent with our previous results on the lower temperature variations for small bodies.

Figure 4 shows the orbit-averaged transverse component $S$ of the seasonal Yarkovsky acceleration, now scaled by the product $R^{\prime} \rho$, as a function of the body's radius $R^{\prime}$. We are

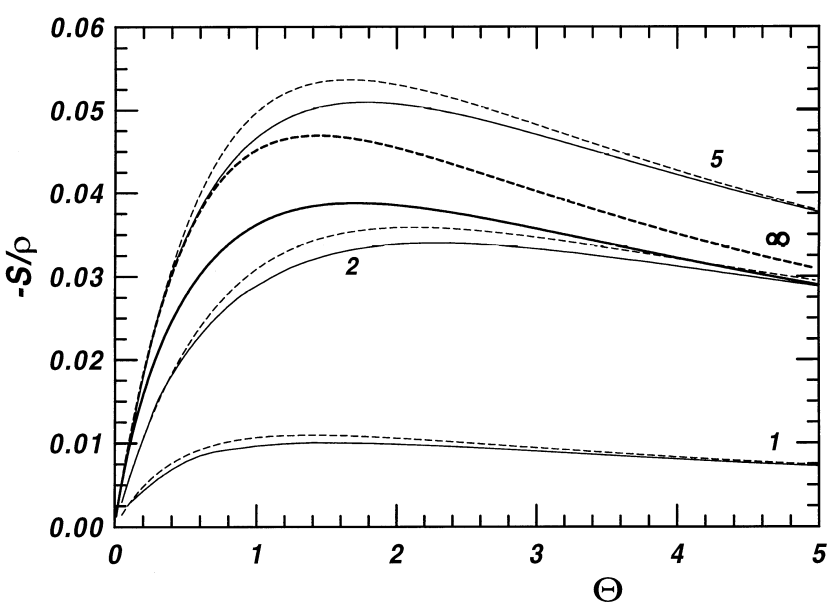

Fig. 3.- Orbit-averaged transverse component $S$ of the seasonal Yarkovsky force per unit mass along a circular orbit as a function of the thermal parameter, $\Theta$. Here $S$ is normalized by the radiation force factor $\rho=\pi R^{2} \mathscr{E}_{*} / m c$ (see text). Bodies of different normalized radii, indicated by labels on the curves, are compared. The dashed curves correspond to the linear solution (eq. [B15]), while the solid curves have been derived by our more accurate numerical approach. The maximum discrepancy, about $20 \%$, is observed for large bodies (labeled $\infty$ ), namely, for the planeparallel solution. 


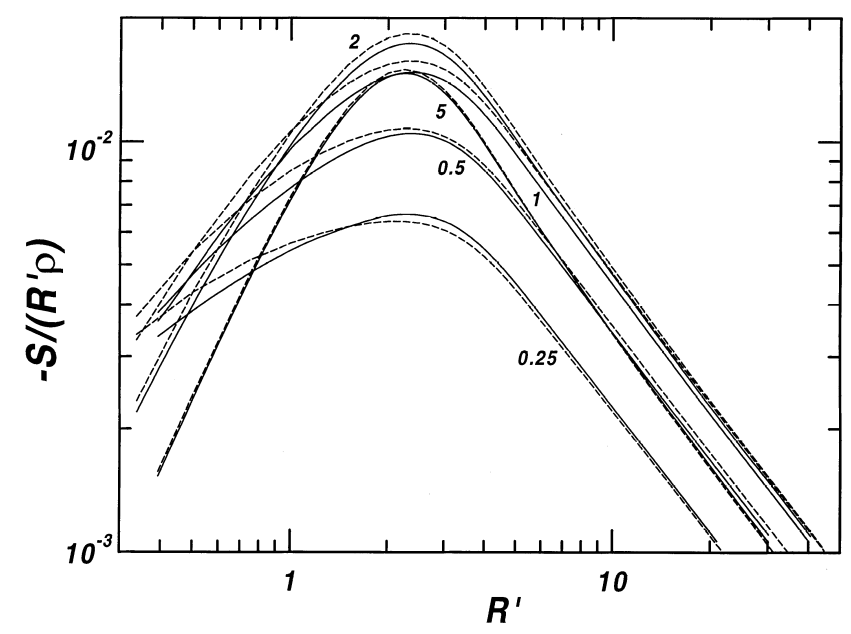

FIG. 4.-Orbit-averaged transverse component $S$ of the seasonal Yarkovsky acceleration (now scaled by the radiation force parameter $\rho$ times the normalized radius $R^{\prime}$ ) vs. $R^{\prime}$. Homogeneous bodies at the same orbital distance satisfy $R^{\prime} \rho \approx$ const. Here the labels of the curves correspond to different values of the thermal parameter, $\Theta$. Results from the complete nonlinear model (solid curves) are compared with those from the linear approximation (dashed curves).

still assuming a circular orbit. Because for homogeneous bodies $\rho \propto R^{-1}$, the product $R^{\prime} \rho$ is a function of the thermal constants and the orbital radius $a$ but not of the body's size. We have chosen a range of values of the thermal parameter $\Theta$ from 0.25 to 5 . To understand the information provided by this figure, we recall that the following values of $\Theta$ and $R$ are relevant for realistic materials: $\Theta=0.323 a^{3 / 4}$ and $R=2.36 R^{\prime} a^{3 / 4}$ for stony bodies; $\Theta=1.627 a^{3 / 4}$ and $R=7.07 R^{\prime} a^{3 / 4}$ for metal-rich bodies. Here we have used the thermal constants adopted by Farinella et al. (1998) and assumed that the surface is not covered by a poorly conducting regolith layer; as discussed in that paper, a plausible estimate for the uncertainty in the thermal parameters corresponds to an uncertainty of about a factor of 2 in the constants given above. Considering objects in the main asteroid belt ( $a$ between 2 and $3 \mathrm{AU}$ ), the thermal parameter may range between 0.5 and 1 for stones and between 2.5 and 4 for metal-rich fragments.

Figure 4 shows an interesting result. Curves corresponding to higher values of $\Theta$ (i.e., irons) exhibit sharper peaks, implying that the maximum efficiency of the draglike effect occurs in a rather limited interval of fragment sizes. On the contrary, curves corresponding to lower values of $\Theta$ (i.e., stones) have a relatively flat plateau at small radii, showing that the orbit decay affects a much broader range of objects. This fact may have important consequences for the delivery of asteroid fragments and the abundance of different types of bodies in the Earth's vicinity.

We also note a discrepancy between the nonlinear theory and its linearized counterpart for small bodies $\left(R^{\prime} \leq 1\right)$. Clearly, this effect has to do with both radial and latitudinal heat conduction that tends to equilibrate the temperature very efficiently on small bodies. The most important northsouth (latitudinal) temperature asymmetry, which causes the seasonal thermal force, is rapidly diminished in the nonlinearized theory, and therefore, the estimated semimajoraxis decay rate becomes somewhat smaller. On the other hand, we note that the nonlinearized theory yields a slightly larger Yarkovsky effect for small values of $\Theta(=0.25)$ and large bodies $\left(R^{\prime} \geq 1\right)$.

\subsection{Eccentric Orbits}

So far only circular orbits have been considered. However, as far as the main applications of the theory to real solar system objects are concerned, it is important to assess how the results described above are modified for orbits with a significant eccentricity. We refer to equation (B13) in Appendix B for the linear-theory predictions on the Yarkovsky force for eccentric orbits.

Let us start by computing the polar temperatures for a spherical body during one revolution around the Sun. As an example, we consider a body with $R^{\prime}=5$, with $\Theta=1$ (approximately corresponding to a $35 \mathrm{~m}$ stone near the outer edge of the asteroid belt), and with spin-axis projections onto the orbit-related unit vectors $\boldsymbol{P}$ and $\boldsymbol{Q}$ given by $s_{P}=s_{Q}=-1 /(2)^{1 / 2}$. The spin axis is thus lying again in the orbital plane. Figure 5 shows the two polar temperatures as a function of the true anomaly $\phi$. As expected, the two temperatures are just shifted by half a revolution in the case of a circular orbit. Moreover, the amplitude of their variation about the mean temperature $\bar{T}^{\prime} \approx 1 /(2)^{1 / 2}$ is relatively small. The basic assumptions of the linearized theory given in Appendix B are rather well satisfied in this case.

However, we remark that as the orbital eccentricity grows, the amplitude of the temperature changes increases in a very significant way. This behavior is clearly due to the large variations of the heliocentric distance during one orbit, resulting in a highly variable incident radiation flux. In the case with 0.6 eccentricity, $\Delta T^{\prime}$ at the north pole is nearly of the same order as the mean temperature $\bar{T}^{\prime}$. Therefore, it is not surprising that for such orbits the linear theory fails to predict accurately the orbital perturbations. Note also that the polar temperature profiles are very different with respect to the zero-eccentricity case. As a result of Kepler's second law, the north pole becomes aligned with

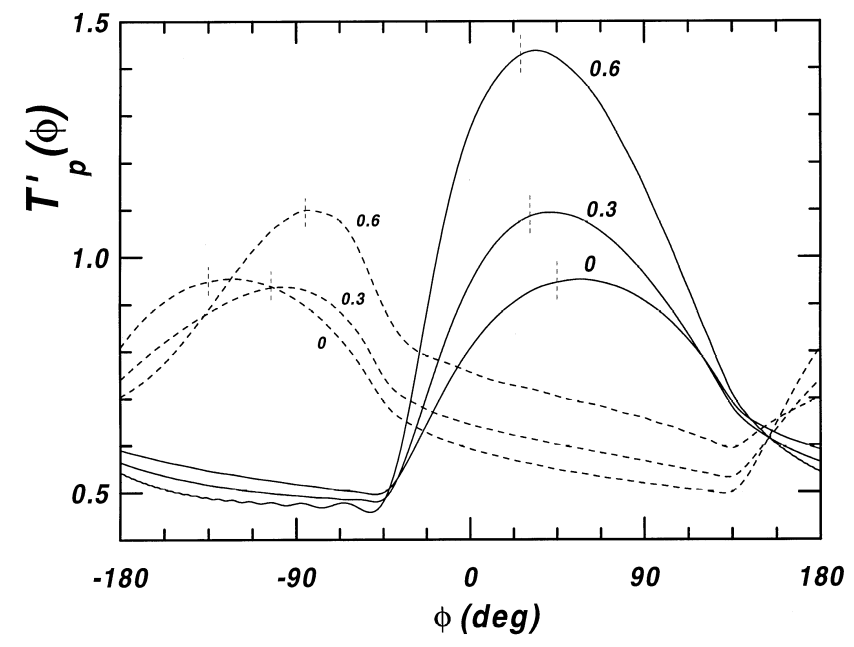

Fig. 5.-Polar temperatures, $T_{p}^{\prime}$, on a spherical fragment vs. the true anomaly, $\phi$, along one orbital revolution: solid curves, behavior of the north $(\mu=1)$ pole temperature; dashed curves, south $(\mu=-1)$ pole temperature. Three orbits with different eccentricities $(0,0.3$, and 0.6$)$ are considered here. Note the strong increase in the amplitude of the temperature variations with the eccentricity and the asymmetry between the polar temperatures. The dashed vertical bars mark the instant of maximum pole illumination for the highest eccentricity orbit. 
the radiation source after the body has passed the pericenter; the south pole, after passing the apocenter. Thus, the maximum radiation fluxes at the poles are quite different, leading to different temperature profiles. In Figure 5, the instants of maximum pole illumination by sunlight are marked by dashed vertical bars. In all these cases, the temperature maximum lags behind the radiation flux, as expected intuitively and as predicted by the linear theory, but interestingly this lag decreases for increasing eccentricities of the orbit. The north pole maximum temperature $(\approx 1.44)$ is smaller than the subsolar temperature at the maximum radiation flux $(\approx 1.55)$, which is another effect of the "thermal memory." In relative terms, this effect is quantitatively about the same as in the circular-orbit case (see Fig. 1, where the maximum temperature does not attain unity but remains smaller).

As for the wavy pattern of the north pole temperature for the 0.6 eccentricity orbit before pericenter passage, this is an artifact of our numerical method, caused by truncation of the Fourier series in equations (10) and (15). In the current example, we took $k_{\max }=34$, but still this order is not high enough for an accurate derivation of the insolation coefficients in a high-eccentricity orbit. Clearly, it is a drawback of our numerical method that facing this problem just by increasing $k_{\max }$ would require rapidly growing computation times. However, since the Yarkovsky force is obtained by an integration of the temperature distribution over the entire surface, it is not affected in a significant way by this problem. The secular effects, derived by averaging over one orbital revolution, should be even less sensitive.

Figure 6 confirms that the integral factor $\int d \mu \mu T^{4}(\mu)$ appearing in the seasonal force in equation (22) does not suffer from this convergence problem. For a comparison, we have plotted here the factor computed from the nonlinear theory (solid lines) and its linear-theory counterpart (dashed lines). The agreement is sufficiently good for the circular orbit; however, as the eccentricity increases we observe a growing discrepancy. This disagreement is not due to a low-eccentricity expansion of the linear theory. Actually, equation (B13) includes all the elliptic expansion terms.

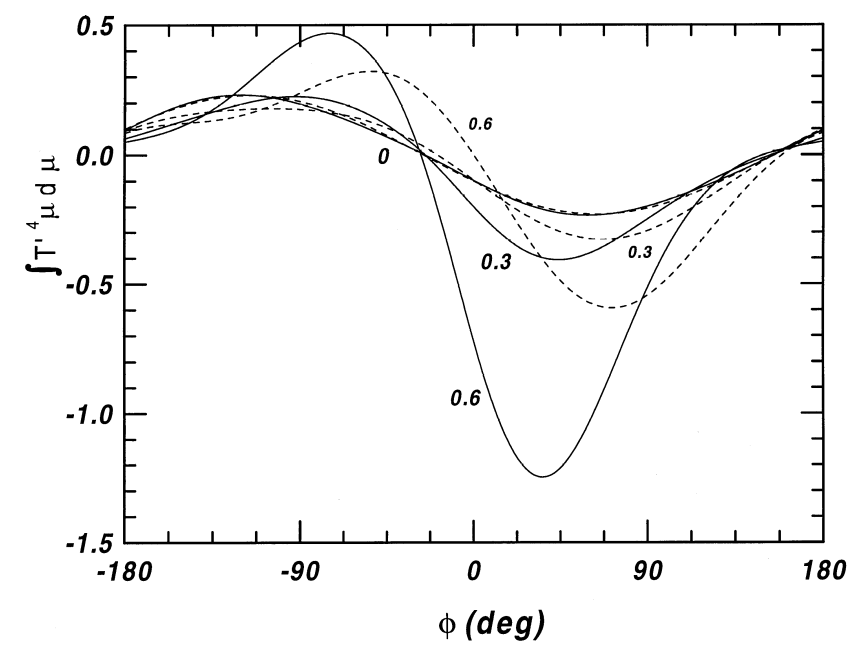

FIG. 6.-The integral factor $\int d \mu \mu T^{\prime 4}(\mu)$ of the seasonal Yarkovsky acceleration for the orbits of Fig. 5 vs. the true anomaly, $\phi$ : solid curves, results from the complete nonlinear theory; dashed curves, from the linear approximation.
Rather, the difference is due to the fact that the basic assumptions of the linear theory cease to be valid for eccentric orbits, as discussed above. The results for the 0.3 eccentricity orbit are already quite off the linear-theory predictions. This seems to be a rather negative finding for a prospect of using the linear theory of the seasonal effect for analytical orbit predictions. However, we shall demonstrate below that the mean drift rate of the semimajor axis, which is related to an average of the integral quantity shown in Figure 6, is much less affected by these nonlinearity effects. This is, in turn, a positive result, indicating that the lineartheory predictions of the orbit-averaged rates of mean elements may be used up to fairly large eccentricities $(\approx 0.5)$ with some degree of confidence.

Figures 7 and 8 show the secular (mean) semimajor-axis drift rate $(d a / d t)$ (scaled by the factor $\left.-\rho R^{\prime} / n\right)$ and the secular eccentricity drift rate $(d e / d t)$ (with the corresponding

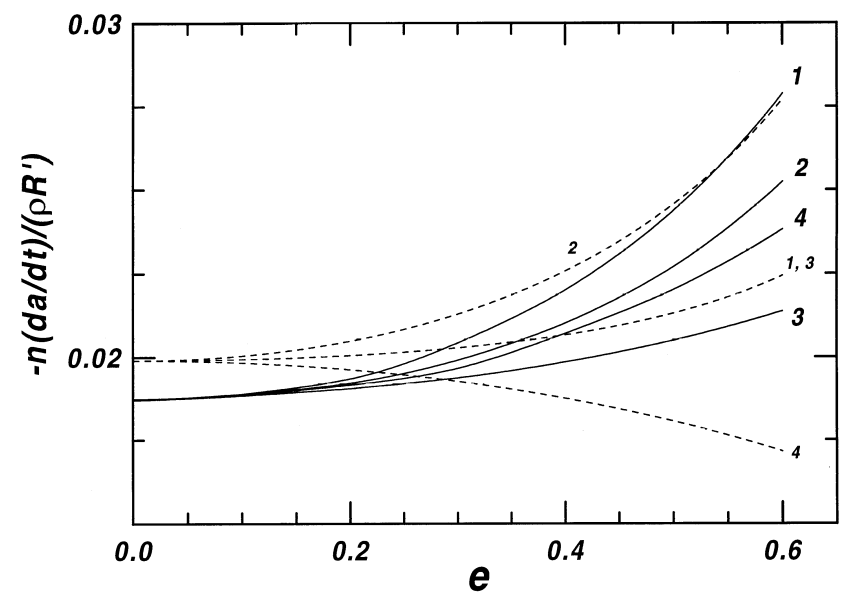

FIG. 7.- Semimajor-axis secular drift rate $(d a / d t)$ (scaled by the factor $\left.-\rho R^{\prime} / n\right)$ vs. eccentricity $e$. Parameter values $R^{\prime}=5$ and $\Theta=1$ have been selected. The different curves correspond to four orientations of the spin axis on the orbital plane, corresponding to the following projections on the $\boldsymbol{P}$ and $\boldsymbol{Q}$ unit vectors: (1) $s_{P}=-1 / \sqrt{2}$ and $s_{Q}=1 / \sqrt{2} ;$ (2) $s_{P}=0$ and $s_{Q}=1$; (3) $s_{P}=s_{Q}=1 / \sqrt{2}$; and (4) $s_{P}=1$ and $s_{Q}=0$. The solid curves have been derived from the complete nonlinear solution; the dashed ones, from the linear theory.

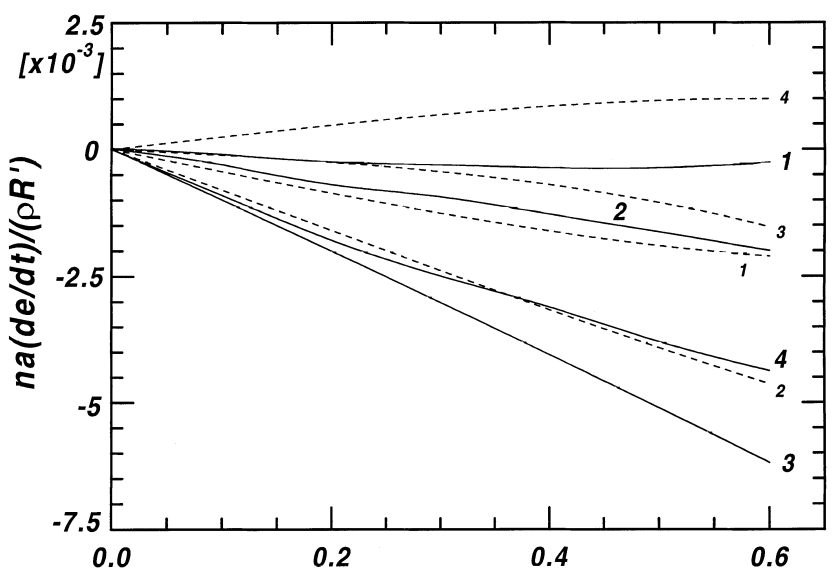

FIG. 8.- Same as Fig. 7, but for the eccentricity secular rate $(d e / d t)$ (scaled by the factor $\rho R^{\prime} / n a$ ). 
scaling factor), computed for orbits with increasing eccentricity. To maximize the seasonal effect, we have selected again the spin axis on the orbital plane, but since for elliptic orbits its orientation with respect to the pericenter direction is important, we have chosen four different orientations, at $45^{\circ}$ intervals. As usual, dashed and solid curves refer to linear-theory (eq. [B16] and [B17] in Appendix B) and nonlinear numerical results, respectively. As the eccentricity of the orbit grows, we observe again a moderately increasing discrepancy between the two approaches, for the reason discussed above. However, the most interesting finding here concerns the fact that the difference between the linearized and nonlinearized theories remains relatively small up to $e \approx 0.4-0.5$ (especially in the semimajor-axis case). This is an unexpected result, since the variables discussed previously (polar temperature, the integral quantity from Fig. 6) exhibit much greater differences when the linear and nonlinear theories are compared.

\section{CONCLUSIONS}

The main results of this paper can be summarized as follows:

1. A nonlinear, numerical solution of the heat transfer problem in a rapidly rotating spherical body has been developed and compared with the corresponding solution from a linearized analytical theory. Assuming constant values of the thermal parameters (thermal conductivity and specific heat), our method does not require solving the heat conduction partial differential equation on a grid. Rather, we use a complete analytic solution of the Fourier equation in terms of a mixed Fourier-Legendre series, and then we iteratively solve the nonlinear boundary constraint.
2. For circular orbits, we estimate that the accuracy of the results from the linear theory for the semimajor-axis decay rate is at worst $20 \%$ (for large bodies with $\Theta \approx 1$ ).

3. The perturbations of all the orbital elements due to the seasonal Yarkovsky effect in a linearized heat transfer theory have been derived analytically to any order in the eccentricity (see Appendix B). In particular, we have proved that to any order in the eccentricity the semimajor axis undergoes a secular decay, so that the orbit shrinks toward the radiation source.

4. As the orbital eccentricity increases, however, the linearized theory fails to match closely the results of the numerical nonlinear theory. This disagreement is not due to a truncation in the eccentricity expansion, but rather to the key assumption of the linear theory that the temperature variations about its mean value stay small. This is already no longer the case at eccentricities $\approx 0.2-0.3$.

5. However, our results suggest that the linear approach is still capable of predicting fairly well the orbit-averaged, long-term changes of the mean elements, in particular the semimajor axis, for orbits up to $e \approx 0.4-0.5$. This is an interesting finding, since the analytic results of the linearized theory might be used to develop semianalytic secular perturbation theories.

We are grateful to W. F. Bottke and D. P. Rubincam for useful discussions on the subject of this paper. Partial support from the Czech Grant Agency (under contract 205/ 96/K119), the Italian Space Agency (ASI), and the Italian Ministry for University and Scientific Research (MURST) is also gratefully acknowledged.

\section{APPENDIX A}

\section{NUMERICAL SOLUTION OF THE NONLINEARIZED PROBLEM}

In this appendix, we briefly comment on the iteration scheme that we developed to solve equation (18). As noted in $\S 2$, we start the iterations with the linearized solution in equations (20), (21a), and (21b). Then, the following rearrangement of the last two equations of the system eqs. ([18a]-[18b]) is used:

$$
\begin{aligned}
& C_{0 l}^{n+1}=\frac{R^{\prime}}{\Theta l}\left[\varepsilon_{0 l}-\sigma_{0 l}\left(C_{p q}^{n}\right)\right], \\
& C_{k l}^{n+1}=\frac{R^{\prime}}{\Theta \psi_{l}\left(Z_{k}\right)}\left[\varepsilon_{k l}-\sigma_{k l}\left(C_{p q}^{n}\right)\right],
\end{aligned}
$$

where $n(\geq 1)$ is the integer label of the iteration ( $k \neq 0$ in eq. [A1b]). The coefficients $\sigma_{k l}$ of the fourth power of the temperature representation (eq. [17]) are computed by numerical quadrature of equations (16a) and (16b) by using Romberg's method. The same method is used to compute the coefficients $\varepsilon_{k l}$ of the energy source function $\mathscr{E}(\mu ; \zeta)$.

To prevent "overshooting" in the subsequent steps of the iteration procedure, especially when $\Theta$ is small and/or $R^{\prime}$ is large, we use the results from equations (A1a) and (A1b) to define a "gradient" of the $C$ coefficients and we perform only a fractional step along this gradient. The value of this fraction depends on the value of the thermal parameter $\Theta$, the radius $R^{\prime}$, and the orbital eccentricity $e$, but generally it ranges from 0.005 to 0.5 . Finally, at each iteration we solve for the $C_{00}$ coefficient (the effective mean temperature of the body) by using the equation

$$
\sigma_{00}\left(C_{p q}\right)=\varepsilon_{00} .
$$

About 10 to 100 iterations of this procedure are needed to attain a $10^{-5}$ accuracy level in satisfying the system of equations (18a)-(18b), depending on the orbital eccentricity and the thermal parameter. The maximum degree of the Fourier development is typically 40 in our computations (i.e., $k \leq 40$ ) and the corresponding maximum degree of the Legendre series development is about 20 (i.e., $l \leq 20$ ). 
The evaluation of the $\psi$-functions from equation (19) represents a special problem in our procedure. The relation

$$
\psi_{l}(z)=z \frac{j_{l}^{\prime}(z)}{j_{l}(z)}=l-z \mathrm{CF}(z, l)
$$

has been found to be the most efficient representation, where we have introduced

$$
\mathrm{CF}(z, l)=\frac{1}{(2 l+3 / 2) / z-} \frac{1}{(2 l+5 / 2) / z-} \cdots .
$$

This continued fraction can be evaluated effectively by using the Lentz algorithm described, for instance, in Press et al. (1994).

\section{APPENDIX B}

\section{LINEARIZED SOLUTION}

Here we shall rederive the linear solution of the heat conduction problem in spherical bodies and rewrite it into our system of "natural" units. The motivation for this is twofold: (1) as discussed in $\S 2$, the linear solution can be used as a suitable starting solution for the iteration scheme needed to solve the full nonlinear problem numerically; and (2) it provides analytical formulae for a number of physically significant quantities - the thermal lag angle, the averaged transverse component of the thermal force, the semimajor-axis decay rate, etc.- -which may be compared with the results of the numerical solution and/or conveniently used in analytical perturbation theories. The relevant references are the papers by Rubincam $(1995,1998)$ and Vokrouhlický (1998a, 1999), who derived the corresponding linear solution for the diurnal Yarkovsky effect. With respect to this earlier work, a novel result obtained here is the expansion of all the relevant quantities to be computed to arbitrary powers of the eccentricity, which extends the applicability of the theory beyond near-circular orbits. Also, we give untruncated formulae for the perturbations of the complete set of five mean elements, which may be exploited in semianalytical treatments of asteroid dynamics under the seasonal Yarkovsky effect perturbation.

The linearity of the solution refers to the fact that we linearize the radiative term in equation (9) and thus make the problem much simpler from a mathematical point of view. In this approximation, the temperature in any $\mu$-ring on the body is assumed always to stay close to a mean (constant) value, so that we can write

$$
T^{\prime}\left(r^{\prime}, \mu ; \zeta\right)=\bar{T}^{\prime}+\Delta T^{\prime}\left(r^{\prime}, \mu ; \zeta\right) .
$$

The mean temperature $\bar{T}^{\prime}$ is given by equation (20) and the linearization condition reads $\left|\Delta T^{\prime}\right| \ll \bar{T}^{\prime}$. The temperature change $\Delta T^{\prime}$ then satisfies the heat transfer equation

$$
i r^{\prime 2} \zeta \frac{\partial}{\partial \zeta} \Delta T^{\prime}\left(r^{\prime}, \mu ; \zeta\right)=\Lambda(r, \mu) \Delta T^{\prime}\left(r^{\prime}, \mu ; \zeta\right)
$$

(identical to eq. [7] for $T^{\prime}$ itself, since $\bar{T}^{\prime}$ is constant), with the linearized boundary condition

$$
\sqrt{2} \eta^{-3 / 4} \Delta T^{\prime}\left(R^{\prime}, \mu ; \zeta\right)+\Theta\left(\frac{\partial \Delta T^{\prime}\left(r^{\prime}, \mu ; \zeta\right)}{\partial r^{\prime}}\right)_{R^{\prime}}=\Delta \mathscr{E}^{\prime}(\mu ; \zeta)
$$

at the surface of the body. The energy flux variation $\Delta \mathscr{E}^{\prime}(\mu ; \zeta)$ is defined by

$$
\Delta \mathscr{E}^{\prime}(\mu ; \zeta)=\mathscr{E}^{\prime}(\mu ; \zeta)-\varepsilon_{00},
$$

where the $\varepsilon_{00}$ term $\left(\varepsilon_{00}=1 /(4 \eta)\right.$; recall that $\eta=\left(1-e^{2}\right)^{1 / 2}$ with $e$ being the eccentricity) is the same appearing in the mean temperature $\bar{T}^{\prime}$. To improve with respect to the results of Rubincam $(1995,1998)$, we shall not neglect the higher order eccentricity terms. Thus, we keep all the harmonics of the Fourier-Legendre representation

$$
\begin{aligned}
\Delta T^{\prime}\left(r^{\prime}, \mu ; \zeta\right) & =\sum_{k \in \mathbf{Z}} \delta t_{k}\left(r^{\prime}, \mu\right) \zeta^{k} \\
& =\sum_{k \in \mathbf{Z}} \sum_{l \geq 0} \delta \tau_{k l}\left(r^{\prime}\right) P_{l}(\mu) \zeta^{k} .
\end{aligned}
$$

Substituting equation (B5) into equation (B2), we easily find that the amplitudes $\delta \tau_{k l}\left(r^{\prime}\right)$ satisfy a system of decoupled elliptic Bessel equations with a general solution given in the following form:

$$
\delta \tau_{n k}\left(r^{\prime}\right)= \begin{cases}\bar{c}_{0 l} r^{\prime l} & \text { for } k=0 \\ \bar{c}_{k l} j_{l}\left(\sqrt{-i k} r^{\prime}\right) & \text { for } k \neq 0\end{cases}
$$

The integration constants $\bar{c}_{k l}$ are to be determined from the boundary constraint (eq. [B3]) and thus are different from the corresponding constants $c_{k l}$ (despite this fact, they can be used as suitable first-iteration seeds for obtaining the exact values of $c_{k l}$ ). The key step then consists in handling the energy influx term in the right-hand side of equation (B3). Rewriting equation (15) as a series of Legendre polynomials, we get

$$
\Delta \mathscr{E}^{\prime}=\text { periodic terms }+\sum_{l \geq 1} \hat{\varepsilon}_{l}(\zeta) P_{l}(\mu),
$$


and we look for a sufficiently simple Fourier expansion of the amplitudes $\hat{\varepsilon}_{l}(\zeta)$. The dipole $(l=1)$ coefficient is the most important as concerns the evaluation of the thermal force in this linearized approximation. Vokrouhlický (1998, unpublished notes; 1999) showed that

$$
\begin{aligned}
\hat{\varepsilon}_{1}(\zeta) & =\frac{1}{2}(a / r)^{2} \cos \theta_{0} \\
& =-\frac{1}{4} \sum_{k \geq 1}\left[\left(s_{P} \alpha_{k}-i s_{Q} \beta_{k}\right) \zeta^{k}+\text { c.c. }\right]
\end{aligned}
$$

(where c.c. stands for the complex conjugate part of the previous expression), with

$$
\alpha_{k}=2 \frac{d}{d e} J_{k}(k e), \quad \beta_{k}=2 \frac{\eta}{e} k J_{k}(k e) .
$$

Here $J_{k}(x)$ are the ordinary Bessel functions of the first order. Explicit expressions of the first seven coefficients $\alpha_{k}$ and $\beta_{k}$ can be found, for instance, in Smart (1953) or Brouwer \& Clemence (1961). We also define for later use the auxiliary factors $\chi_{k} \equiv s_{P} \alpha_{k}-i s_{Q} \beta_{k} \propto e^{k-1}$. The dipole boundary constraint reads

$$
\sqrt{2} \eta^{-3 / 4} \delta \tau_{1 k}\left(R^{\prime}\right)+\Theta\left(\frac{\partial \delta \tau_{1 k}}{\partial r^{\prime}}\right)_{R^{\prime}}=-\frac{1}{4} \chi_{k}
$$

$(k \neq 0)$, leading to

$$
\begin{aligned}
\bar{c}_{k 1} & =-\frac{\chi_{k} \eta^{3 / 4}}{4 \sqrt{2} j_{1}\left(Z_{k}\right)} \frac{1}{1+\lambda \psi_{1}\left(Z_{k}\right)} \\
& =-\frac{\chi_{k} \eta^{3 / 4}}{4 \sqrt{2} j_{1}\left(Z_{k}\right)(1+\lambda)} \frac{A\left(X_{k}\right)+i B\left(X_{k}\right)}{C\left(X_{k}\right)+i D\left(X_{k}\right)} .
\end{aligned}
$$

Here $\psi_{1}\left(Z_{k}\right)$ is the same function as in equation (19). The following auxiliary quantities have been introduced: $X_{k}=(2 k)^{1 / 2} R^{\prime}$, $\lambda=\Theta \eta^{3 / 4} / X_{1}$, whereas the functions $A, B, C$, and $D$ are defined by

$$
\begin{aligned}
& A\left(X_{k}\right)=-\left(X_{k}+2\right)-e^{X_{k}}\left[\left(X_{k}-2\right) \cos X_{k}-X_{k} \sin X_{k}\right], \\
& B\left(X_{k}\right)=-X_{k}-e^{X_{k}}\left[X_{k} \cos X_{k}+\left(X_{k}-2\right) \sin X_{k}\right], \\
& C\left(X_{k}\right)=A\left(X_{k}\right)+\frac{\lambda}{1+\lambda}\left\{3\left(X_{k}+2\right)+e^{X_{k}}\left[3\left(X_{k}-2\right) \cos X_{k}+X_{k}\left(X_{k}-3\right) \sin X_{k}\right]\right\}, \\
& D\left(X_{k}\right)=B\left(X_{k}\right)+\frac{\lambda}{1+\lambda}\left\{X_{k}\left(X_{k}+3\right)-e^{X_{k}}\left[X_{k}\left(X_{k}-3\right) \cos X_{k}-3\left(X_{k}-2\right) \sin X_{k}\right]\right\} .
\end{aligned}
$$

Given the similarity of the representations in equations (B5) and (10) for the temperature distribution in the linearized and the nonlinearized approaches, we may use $C_{00} \approx \bar{T}^{\prime}$ and $C_{k 1} \approx \bar{c}_{k 1} j_{1}\left(Z_{k}\right)$ for the initial estimate in our iteration scheme in Appendix A. In principle, one might also compute the linearized estimate of the quadrupole and higher multipole coefficients $\bar{c}_{2 k}, \ldots$, obtaining thus linear-theory initial guesses for the coefficients $C_{k 2}, \ldots$, but this is not needed in practice.

Putting all the previous results together, we easily find the dipole part of the expansion of the temperature variation in equation (B5). With the surface temperature variation $\Delta T^{\prime}$ determined at the dipole level, we may use equation (22) to compute the thermal force. In the linearized case, for the $T^{\prime 4}$ term in the integrand we substitute (2) $\eta^{-3 / 4} \Delta T^{\prime}$. As expected, the force per unit mass $a_{\mathrm{th}}$ is aligned with the spin axis unit vector $s$, namely, $a_{\mathrm{th}}=a_{\mathrm{th}} \boldsymbol{s}$. We are then concerned with the amplitude $a_{\mathrm{th}}$ of the thermal acceleration. After some algebra one obtains

$$
\begin{aligned}
a_{\mathrm{th}} & =\frac{2}{9} \rho \frac{1-A}{1+\lambda} \sum_{k \geq 1}\left(\chi_{k} G_{k} e^{i \delta_{k}} \zeta^{k}+\text { c.c. }\right) . \\
& =\frac{4}{9} \rho \frac{1-A}{1+\lambda} \sum_{k \geq 1} G_{k}\left[s_{P} \alpha_{k} \cos \left(k n t+\delta_{k}\right)+s_{Q} \beta_{k} \sin \left(k n t+\delta_{k}\right)\right],
\end{aligned}
$$

with the thermal lag angles $\delta_{k}$ given by

$$
G_{k} \cos \delta_{k}=\frac{A\left(X_{k}\right) C\left(X_{k}\right)+B\left(X_{k}\right) D\left(X_{k}\right)}{C\left(X_{k}\right)^{2}+D\left(X_{k}\right)^{2}}, \quad G_{k} \sin \delta_{k}=\frac{B\left(X_{k}\right) C\left(X_{k}\right)-A\left(X_{k}\right) D\left(X_{k}\right)}{C\left(X_{k}\right)^{2}+D\left(X_{k}\right)^{2}},
$$

( $\rho$ is defined as in eq. [22]). The acceleration (eq. [B13]) can be used both for numerical integrations (as in Brož 1999) and in analytical perturbation theories. A detailed discussion of these applications is beyond the scope of this paper, and we shall limit ourselves to a couple of specific comments.

The most important orbital effect of the seasonal Yarkovsky force in equation (B13) is that it gives rise to a secular semimajor-axis decay. A simple estimate can be given for a near-circular orbit. In this case, the secular rate of change of the 
semimajor axis is given by $d a / d t=2 S / n+O(e)$, where $S$ is the orbit-averaged value of the transverse component of the thermal force. Some algebra leads to

$$
S=\frac{2}{9}(1-A) \rho \frac{G_{1} \sin \delta_{1}}{1+\lambda} \chi_{1} \bar{\chi}_{1}+O(e) .
$$

Note the geometric factor $\chi_{1} \bar{\chi}_{1}=s_{P}^{2}+s_{Q}^{2}=\sin ^{2} \gamma$, where $\gamma$ is the obliquity of the spin axis with respect to the normal to the orbital plane (compare with the results reported by Rubincam 1987, 1995 and Farinella et al. 1998). Equation (B15) is equivalent to the corresponding result of Rubincam (1998). As the thermal response always lags behind the external heating, all the angles $\delta_{k}$ are negative (this can be easily shown using equations [B12a]-[B12d]). Since all the other factors in equation (B15) are positive, including the geometric factor $\chi_{1} \bar{\chi}_{1}=\sin ^{2} \gamma$, one verifies that $d a / d t<0$ for any orientation of the spin axis.

Let us now discuss the corrections to the previous estimate of the orbital decay rate due to the eccentricity terms. The related algebra becomes quite cumbersome because of the increasing importance of the higher order Fourier terms in equation (B13). However, as a rule of thumb, $\chi_{k} \propto e^{k-1}$, and this allows us to estimate the order of magnitude of the contribution of any term of a given order. Luckily, when the secular perturbations in the orbital elements are concerned, this decrease for growing eccentricities is accelerated. Carrying out the calculation to an arbitrary order in the eccentricity we obtain

$$
\frac{d a}{d t}=\frac{4}{9 n} \rho \frac{1-A}{1+\lambda} \sum_{k \geq 1} \frac{G_{k}}{k} \sin \delta_{k} \chi_{k} \bar{\chi}_{k} .
$$

Again the universal semimajor-axis decrease can be verified, since all the lag angles $\delta_{k}$ are negative and the geometric factors $\chi_{k} \bar{\chi}_{k}=s_{P}^{2} \alpha_{k}^{2}+s_{Q}^{2} \beta_{k}^{2}$ are always positive. Note also that the eccentricity factors $\alpha_{k}$ and $\beta_{k}$ thus appear quadratically in equation (B16). This property generates an accelerated convergence of this series when compared with the instantaneous-force formula (eq. [B13]) and may be the reason why the nonlinearized theory yields a rather good prediction for the long-term semimajor-axis decay (see Fig. 7 and related discussion). Another consequence is that the secular semimajor-axis decay based on the formula in equation (B15) is actually correct to the second order in the eccentricity.

The case of the secular perturbation of the eccentricity is slightly more complicated (see discussion in Rubincam 1995, 1998; Vokrouhlický \& Farinella 1998b). For instance, Rubincam $(1995,1998)$ was unable to give a sufficiently simple expression for this perturbation. As in the semimajor-axis case, our compact formulation allows us to obtain fairly general results. Choosing as a variable $p=a \eta^{2}$ instead of the eccentricity, it can be shown that a complete formula for its secular change due to the seasonal Yarkovsky effect reads

$$
\frac{d p}{d t}=\frac{4 \eta}{9 n} \rho \frac{1-A}{1+\lambda} \sum_{k \geq 1} \frac{G_{k}}{k^{2}}\left[\alpha_{k} \beta_{k} \sin \delta_{k} \sin ^{2} \gamma+s_{P} s_{Q}\left(\alpha_{k}^{2}-\beta_{k}^{2}\right) \cos \delta_{k}\right] .
$$

Working to zeroth order in the eccentricity, we easily verify that $d p / d t=d a / d t+O\left(e^{2}\right)$, thanks again to the quadratic appearance of the $\alpha_{k}$ and $\beta_{k}$ factors in the right-hand side of equation (B17). As a consequence, de/dt $\propto O(e)$. The correct proportionality factor can be obtained from a combination of equations (B16) and (B17).

The secular perturbations of the inclination $I$ and the longitude of the ascending node $\Omega$ can be given in the form

$$
\begin{aligned}
& \frac{d \Omega}{d t} \sin I \cos \omega-\frac{d I}{d t} \sin \omega=\frac{2}{9} \rho \frac{1-A}{1+\lambda} \frac{\cos \gamma}{n a \eta} \sum_{k \geq 1} \frac{G_{k}}{k^{2}} \beta_{k}\left(s_{Q} \beta_{k} \cos \delta_{k}-s_{P} \alpha_{k} \sin \delta_{k}\right), \\
& \frac{d \Omega}{d t} \sin I \sin \omega+\frac{d I}{d t} \cos \omega=\frac{2}{9} \rho \frac{1-A}{1+\lambda} \frac{\cos \gamma}{n a \eta} \sum_{k \geq 1} \frac{G_{k}}{k^{2}} \alpha_{k}\left(s_{Q} \beta_{k} \sin \delta_{k}+s_{P} \alpha_{k} \cos \delta_{k}\right) .
\end{aligned}
$$

As before, no truncation in the eccentricity has been performed here.

Finally, the untruncated mean drift of the pericenter $\omega$ is obtained in the form

$$
\frac{d \omega}{d t}+\sin I \frac{d \Omega}{d t}=\frac{2}{9 n} \rho \frac{1-A}{1+\lambda} \sum_{k \geq 1} \frac{G_{k}}{k^{2}}\left(-s_{P} s_{Q} \hat{\alpha}_{k} \sin \delta_{k}+\hat{\beta}_{k} \cos \delta_{k}\right),
$$

where we have defined

$$
e \hat{\alpha}_{k}=k \eta^{3}\left(\alpha_{k}^{2}-\beta_{k}^{2}\right)-e^{2} \alpha_{k} \beta_{k}, \quad e \hat{\beta}_{k}=k \eta^{3} \alpha_{k} \beta_{k} \sin ^{2} \gamma-\left(s_{Q}^{2} \beta_{k}^{2}+s_{P}^{2} \alpha_{k}^{2}\right) .
$$

Interestingly, one easily verifies that the mean drift of the pericenter given by equation (B19), like in the eccentricity case (eq. [B17]), is not singular at zero eccentricity.

Equations (B16)-(B19) derived above yield the secular rates of change of the mean orbital elements for arbitrarily eccentric orbits. They can be used for semianalytical investigations of asteroid dynamics including the seasonal Yarkovsky effect. A detailed study of the interaction between Yarkovsky perturbations and the mean motion resonances in the main asteroid belt can be mentioned here as a particularly important problem (see, e.g., Bottke, Rubincam, \& Burns 1999; Brož 1999; Brož et al. 1999). 


\section{REFERENCES}

Bottke, W. F., Rubincam, D. P., \& Burns, J. A. 1999, Icarus, in press

Brouwer, D., \& Clemence, G. 1961, Methods of Celestial Mechanics (New York: Academic)

Brož, M. 1999, Diploma thesis, Charles Univ.

Brož, M., Vokrouhlický, D., Farinella, P., \& Bottke, W. F. 1999, BAAS, 31, 1111

Burns, J. A., Lamy, P. L., \& Soter, S. 1979, Icarus, 40, 1

Farinella, P., \& Vokrouhlický, D. 1999, Science, 283, 1507

Farinella, P., Vokrouhlický, D., \& Hartmann, W. K. 1998, Icarus, 132, 378

Öpik, E. J. 1951, Proc. R. Irish Acad., 54, 1650

Peterson, C. 1976, Icarus, 29, 91

Press, W. H., Flannery, B. P., Teukolsky, S. A., \& Vetterling, W. T. 1994

Numerical Recipes (Cambridge: Cambridge Univ. Press),

Radzievskii, V. V. 1952, AZh, 29, 162
Rubincam, D. P. 1987, J. Geophys. Res., 92, 1287

. 1994, Theor. Appl. Climatol., 48, 195

1995, J. Geophys. Res., 100, 1585

1998, J. Geophys. Res., 103, 1725

Smart, W. M. 1953, Celestial Mechanics (London: Longmans)

Spencer, J. R., Lebofsky, L. A., \& Sykes, M. V. 1989, Icarus, 78, 337

Vokrouhlický, D. 1998, A\&A, 335, 1093 1999, A\&A, 344, 362

Vokrouhlický, D., \& Brož, M. 1999, A\&A, 350, 1079

Vokrouhlický, D., \& Farinella, P. 1997, Planet. Space Sci., 45, 419 1998a, A\&A, 335, 351

1998 b, AJ, 116, 2032

1999, Nature, submitted 